\title{
Cost-Benefit Study of Febrile Neutropenia Prophylactic Treatment with Pegfilgrastim vs. Filgrastim in Pediatric Patients with Solid Tumors
}

\author{
Norma Araceli López-Facundo ${ }^{1, *}$, Ma. Guadalupe Valois-Escamilla ${ }^{2}$, Isidoro Tejocote-Romero \\ Cecilia Rodríguez-Castillejos ${ }^{3}$ and Yanet Jaimes-García ${ }^{1}$
}

${ }^{1}$ Department of Pediatric Oncology, 2 Pediatrics Department and ${ }^{3}$ Department of Pediatric Hematology; Hospital Materno-Infantil, ISSEMYM, Toluca, Estado de México, Mexico

Received for publication: 6 December 2016; accepted for publication: 25 February 2017

Available online: 14 July 2017

\section{KEYWORDS \\ Cost-benefit; \\ Pegfilgrastim; \\ Febrile neutropenia}

\begin{abstract}
Background: Febrile neutropenia is one of the main complications in cancer patients that exponentially increases treatment costs. Pegfilgrastim is a pegylated form of fil= grastim, and it might reduce the severity and duration of febrile neutropenia, as well as costs. Objective: To analyze the cost-benefit ratio of febrile neutropenia prophylactic treatment with pegfilgrastim vs. filgrastim in pediatric patients with solid tumors. Methods: A cost-benefit study was carried out, where complete medical records of pediatric patients with solid tumors and febrile neutropenia who received prophylactic treatment with pegfilgrastim vs. filgrastim were analyzed. Clinical-demographic variables, febrile neutropenia events, and days of hospital stay were considered, as well as related complications and global related cost, looking for differences by means of Student's t-test and the chi-square test. Results: Twenty-six patients with a total of 106 chemotherapy courses were included. Fourteen patients $(52.8 \%)$ were admin $=$ istered filgrastim and 12 patients (46.1\%) received pegfilgrastim. There were $57.6 \%$ of males. Mean hospital days of stay and costs were significantly higher in the filgrastim versus the pegfilgrastim group $(p<0.001)$. Conclusion: The use of pegfilgrastim reduced the number of neutropenia and fever events, days of hospital stay, and costs by up to $30 \%$. (creativecommons.org $/$ licenses/by-nc-nd/4.0/).
\end{abstract}

*E-mail for correspondence: oncouaem2009@hotmail.com (N.A. López-Facundo) 


\section{INTRODUCTION}

Costs associated with febrile neutropenia (FN)-related hospitalization significantly add to the direct medical costs of cancer treatment, and entail a heavy financial burden on these patients' global care ${ }^{1-4}$. Individuals with FN can be treated as in- or outpatients according to the risk for complications ${ }^{5}$. Multiple cost-utility models have been developed in order to compare the economic effects of different therapeutic alternatives. Randomized trials with sargramostim, filgrastim, and pegfilgrastim have been reported, which have shown significantly less FN events and FN-related hospital-stay days and, therefore, lower costs related to these events ${ }^{6}$. In other pharmacoeconomic studies conducted in the USA, a cost decrease has been observed with the use of primary prophylaxis in patients receiving chemotherapy. Among the granulocyte colony-stimulating factor (G-CSF) drugs, pegfilgrastim appears to be superior to filgrastim in terms of cost minimization, and primary prophylaxis seems to be less expensive than secondary prophylaxis. A decrease in the risk of infection-related mortality has been observed, as well as the benefit of being able to maintain the chemotherapy dose intensity with the support of G-CSF agents ${ }^{7-10}$. A cost-utility model based on usual clinical practice for the treatment of FN with immediate hospitalization or ambulatory treatment, developed in the USA, provides robust evidence indicating that pegfilgrastim is not only costeffective but it also generates cost savings in clinical areas by reducing mean hospitalization-day costs estimated in patients who survived a FN event with regard to those who died. At baseline conditions, pegfilgrastim is superior to filgrastim and other G-CSF agents, with expected costs and effectiveness of US\$ 4,203 and 12,361 qualityadjusted life-days (QALD) in case of no G-CSF use, US\$ 3,058 and 12,967 QALDs with pegfilgrastim, and US\$ 5,655 and 12,698 QALDs with the use of filgrastim, which indicates that pegfilgrastim prophylactic administration produces both clinical and economic benefits ${ }^{3-12}$. In the UK, Germany, and Japan, studies with G-CSF agents have been carried out in breast cancer, with pegfilgrastim-associated $\mathrm{FN}$ absolute risk being estimated to be $5.5 \%$ lower than with 10 days on filgrastim (7 vs. $12.5 \%$ ), and $10.5 \%$ lower than with six days on filgrastim (7 vs. $17.5 \%$ ). A very elevated incremental cost-effectiveness ratio per life-year gained was shown, with an up to Euros 2,229 saving being found, and an adjusted gain of 0.039 quality-adjusted life-years (QALY) with the use of primary prophylaxis with pegfilgrastim ${ }^{13-16}$. Cost-efficacy studies of G-CSF agents such as biosimilar filgrastim and pegfilgrastim have been carried out in European countries ${ }^{17}$. Febrile neutropenia prophylaxis or treatment with the biosimilar drug is cost-effective in all possible treatment scenarios with regard to filgrastim and pegfilgrastim ${ }^{18-30}$.

There are few pediatric clinical trials to validate pegfilgrastim safety and effectiveness in children. However, those already reported suggest good tolerance and safety, with few side effects ${ }^{31,32}$.

The purpose of the present study was to assess the cost-benefit ratio of $\mathrm{FN}$ prophylaxis with pegfilgrastim vs. filgrastim in pediatric patients with solid tumors.

\section{MATERIAL AND METHODS}

Medical records of pediatric patients with solid tumors who received at least four courses of myeloablative chemotherapy were included in a cost-benefit study carried out at the Pediatric Oncology Unit of the Mother-Child Hospital of the Institute of Social Security of the State of Mexico and Municipalities. The patients had to have received a single dose of pegfilgrastim (Neulastim ${ }^{\circledR}$ ) of $100 \mathrm{mcg} / \mathrm{kg}$ at day 6 and were compared with a historical cohort of patients who received filgrastim (Inmunef ${ }^{\circledR}$ or Neupogen ${ }^{\circledR}$ ) at $5 \mathrm{mcg} / \mathrm{kg} /$ day from day 6 to 15 during the period of December 2010 through July 2013. Courses where the patients had received radiotherapye simultaneous to chemotherapy or one month prior to chemotherapy were excluded. Clinical-demographic variables such as age, gender, type of tumor, administered chemotherapy scheme, number of FN events requiring hospitalization (with neutropenia being regarded as an absolute neutrophil count $<1,500$ and fever as a temperature $>38.3$ in two occasions or a fever peak > 39 degrees, according to the WHO), blood count at hospital admission, type of infection, presence of FN-related complications, number of hospital days of stay, as well as global costs associated with these events, were assessed. The costs of bed-day, medications including filgrastim extra doses, laboratory and imaging studies, among others, were considered.

The costs were provided and crosschecked by the Accountability Department of the Hospital based on figures published in the Government Gazette. Data were analyzed using the SPSS software, version 17. Descriptive statistics was used with central tendency and dispersion measures, as well as Student's $t$-test and the chi-square test to look for differences in both groups. In addition, hospitalization risk was determined by means of bivariate analysis.

The present study was approved by the Research Ethics Committee of the Hospital.

\section{RESULTS}

The medical records of 26 patients with a total of 106 chemotherapy courses were thoroughly analyzed. There were $57.6 \%$ males and $42.3 \%$ females in the sample, with an average age at first chemotherapy course of $91.4 \pm 66$. 1 months (9-192 months). The main tumors included in this study are described in table 1. Of the 106 chemotherapy courses, pegfilgrastim prophylaxis was administered 52 times in 12 patients $(46.1 \%)$.

The main chemotherapies used in these patients are described in table 1. The following doses were administered:

- ifosfamide $1.8 \mathrm{~g} / \mathrm{m}^{2} \mathrm{SC} \mathrm{d} 1-5$, carboplatin $450 \mathrm{mg} / \mathrm{m}^{2}$ SC d1, etoposide $100 \mathrm{mg} / \mathrm{m}^{2}$ SC d1-5 (ICE);

- vincristine $2 \mathrm{mg} / \mathrm{m}^{2} \mathrm{SC} \mathrm{d} 1$, ifosfamide $1.8 \mathrm{~g} / \mathrm{m}^{2} \mathrm{SC} \mathrm{d} 1-5$, actinomycin D $15 \mathrm{mcg} / \mathrm{kg} \mathrm{d} 1-5$, etoposide $100 \mathrm{mg} / \mathrm{m}^{2}$ SC d1-5 (VAI-VIE);

- vincristine $2 \mathrm{mg} / \mathrm{m}^{2} \mathrm{~d} 1$, Adriamycin $35 \mathrm{mg} / \mathrm{m}^{2} \mathrm{SC} \mathrm{d} 1-2$, cyclophosphamide $2.1 \mathrm{~g} / \mathrm{m}^{2} \mathrm{SC} \mathrm{d} 1-2$ (VAC).

Febrile neutropenia requiring hospitalization occurred in 35 occasions in $9 / 53$ chemotherapy courses receiving prophylactic pegfilgrastim vs. $26 / 53$ courses prophylactically treated with filgrastim. In the bivariate analysis for hospitalization risk in pegfilgrastim-treated patients, an overall risk 
Table 1. Characteristics of pediatric patients with solid tumors who received febrile neutropenia prophylaxis with filgrastim vs. pegfilgrastim

\begin{tabular}{lcc}
\hline & $\begin{array}{c}\text { Filgrastim } \\
(\mathrm{n}=14)\end{array}$ & $\begin{array}{c}\text { Pegfilgrastim } \\
(\mathrm{n}=12)\end{array}$ \\
\hline Age in months & $\begin{array}{c}\text { Mean } 97 \\
(12-204)\end{array}$ & $\begin{array}{c}\text { Mean } 112 \\
(12-192)\end{array}$ \\
Gender: & & \\
- Male & 8 & 7 \\
- Female & 6 & 5 \\
Diagnosis: & & \\
- Rhabdomyosarcoma & 3 & 3 \\
- Germ cell tumors & 3 & 2 \\
- Soft tissue sarcoma & 2 & 2 \\
- Wilms tumor & 1 & 0 \\
- Neuroblastoma & 2 & 2 \\
- Other & 3 & 3 \\
Type of chemotherapy*: & $\mathrm{N} 53$ & $\mathrm{~N} 53$ \\
- ICE & 25 & 27 \\
- VAl-VIE & 24 & 25 \\
- VAC & 4 & 1 \\
\hline
\end{tabular}

*Number of chemotherapy courses.

ICE: ifosfamide/etoposide with or without carboplatin; VAC: vincristine, actinomycin, cyclophosphamide; VAl: vincristine, actinomycin, ifosfamide; VIE: vincristine, ifosfamide, etoposide.

of 0.21 was obtained $(95 \% \mathrm{Cl}: 0.08-0.5 ; \mathrm{p}=0.01)$, indicating that the use of pegfilgrastim significantly decreased the number of hospitalizations. Hospitalization was more commonly observed in males, without the difference reaching statistical significance $(p=0.101)$. With regard to the leukocyte count at the time of FN-related hospital admission, no statistically significant difference was found in leukocyte or total neutrophil counts; however, the monocyte count was not considered at admission. Pegfilgrastim-treated patients were observed to recover leukocyte counts faster than those treated with filgrastim, with the number of blood product transfusions being higher in filgrastim-treated patients. Most patients experienced fever and neutropenia with no evident clinical focus, followed by respiratory tract infections and gastroenteritis, among others. Three patients treated with filgrastim and two with pegfilgrastim were admitted to the intensive-care department due to sepsis and/or shock. Ayerage days of hospital stay was significantly higher in the group of chemotherapy courses that received filgrastim: 5.4 \pm 1.5 vs. $5.4 \pm 1.6$ days in those treated with pegfilgrastim, $\mathrm{p}=0.017$ (Table 2). When the cost of FN events was analyz ed in Mexican pesos in both treatment groups, the chemo therapy courses with filgrastim-based prophylaxis were observed to be a mean of $\$ 16,206.3 \pm 4,531.1$ pesos, whereas in those treated with pegfilgrastim it was $\$ 8,723.8 \pm 3,644$ pesos, with the difference being statistically significant $(p$ $<0.001)$. Global cost included hospitalization days, medi cations, transfusions, laboratory and imaging studies, etc. as well as the costs of the G-CSF drugs (filgrastim cost: $\$$ 1,200 per vial; pegfilgrastim: $\$ 22,000$ per vial; government prices), and we observed that prophylaxis with pegfilgrastim reduced the total cost covered by the institution by up to $25 \%$ approximately in comparison with the use of filgrastim, with total global cost of $\$ 1,018,323$ vs. $1,278,186$, respec tively (Fig. 2). Both groups required support with blood products: 12 hospitalized patients (46.1\%) were transfused red blood cell concentrates and 11 patients (42\%) platelet concentrates, with the difference being that those patients treated with filgrastim had grade 3 or 4 hematologic toxicity ( $\mathrm{Hb} 6.5-8 \mathrm{~g} / \mathrm{dl}$ and platelets $25-50,000 / \mathrm{mm}^{3}$ for grade 3 or $25,000 / \mathrm{mm}^{3}$ for grade 4 ), thus requiring a higher number of red blood cell and platelet concentrates in comparison with those in whom pegfilgrastim was administered. However, it should be noted that this study only included blood product administration costs, without considering the cost related to obtain them (laboratory tests, double serology for each donor, equipment to obtain blood samples, use of laborat tory equipment for each one of the blood products, etc.).

Table 2. Febrile neutropenia prophylaxis results in children with solid tumors treated with filgrastim vs. pegfilgrastim

\begin{tabular}{|c|c|c|c|}
\hline & $\begin{array}{l}\text { Filgrastim } \\
(n=53)\end{array}$ & $\begin{array}{l}\text { Pegfilgrastim } \\
\qquad(\mathrm{n}=53)\end{array}$ & $P$ \\
\hline Febrile neutropenia events* & 26 & 9 & 0.000 \\
\hline Leukocyte count ${ }^{* *}$ & $851(100-1,150) / \mathrm{mm}^{3}$ & $1,066(160-1,240) / \mathrm{mm}^{3}$ & 0.55 \\
\hline Absolute neutrophils ${ }^{\dagger}$ & $204(0-570) / \mathrm{mm}^{3}$ & $208(0-660) / \mathrm{mm}^{3}$ & 0.95 \\
\hline \multicolumn{4}{|l|}{ Type of infection: } \\
\hline - FN without focus & 9 & 2 & \\
\hline - Respiratory tract infection & 7 & 4 & \\
\hline - Gastroenteritis & 6 & 0 & \\
\hline - Other & 8 & 2 & \\
\hline \multicolumn{4}{|l|}{ Hospitalization days: } \\
\hline Average (range) & $5.4(3-9)$ & $3.7(2-6)$ & 0.017 \\
\hline Cost per neutropenia event ${ }^{+}$ & $\$ 16,206.00 \pm 4,531.00$ & $\$ 8,723.00 \pm 3,644.00$ & 0.001 \\
\hline Prophylactic treatment global cost ${ }^{\ddagger}$ & $\$ 1,278,186.00$ & $\$ 1,018,323.00$ & 0.001 \\
\hline
\end{tabular}

${ }^{*}$ Required hospitalization; ${ }^{\dagger}$ At febrile neutropenia-related hospital admission; ${ }^{\ddagger}$ Mexican pesos.

FN: febrile neutropenia. 


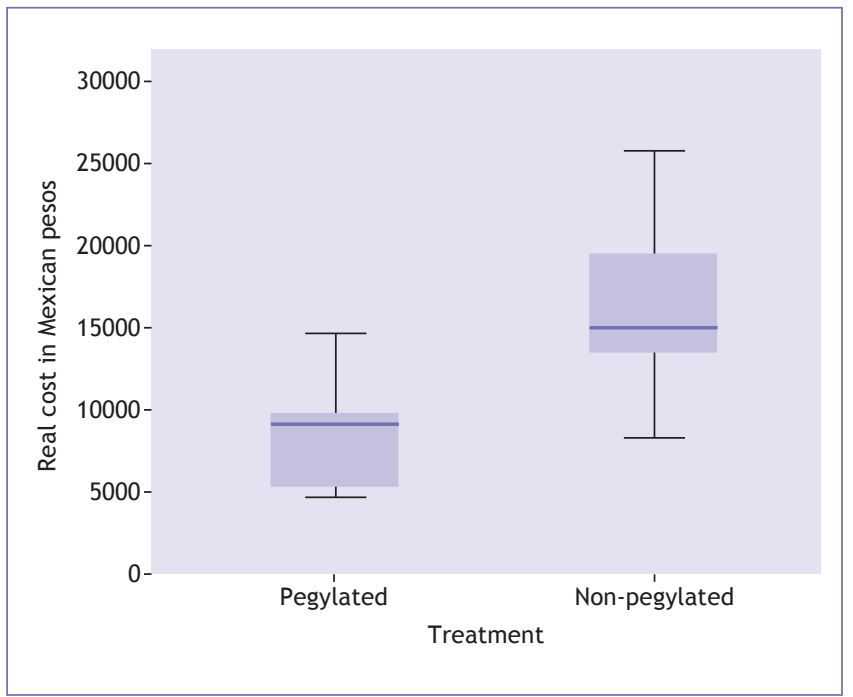

Figure 1. Global cost per febrile neutropenia event in pediatric patients with solid tumors prophylactically treated with pegfilgrastim vs. filgrastim (Mexican pesos).

In addition, in both groups there were patients who required management in the pediatric intensive-care department, which increased the costs owing to the serious condition of the patient, the continuous use of monitors, infusion pump, laboratory and imaging studies, and even parenteral nutrition, among others, with the difference being made by hospital-stay days. Another factor that modified this cost difference is the use of broad spectrum antimicrobial, antifungal, and antiviral agents in the required cases, without taking into account the outpatient use of antimicrobials that did not require hospitalization.

\section{DISCUSSION}

In this cost-benefit study of primary prophylaxis with filgrastim vs. pegfilgrastim in cancer patients treated with myeloablative chemotherapy, only patients with FN episodes warranting hospital admission were considered, without those requiring transfusions or antimicrobial use in the outpatient setting being included. A decrease of $50-60 \%$ in $\mathrm{FN}$ events with the prophylactic use of G-CSF is reported in the international literature ${ }^{1,14}$. During the studied period, hospitalization was required in $33 \%$ of all courses. In the study by Eldar-Lissai, et al. the use of pegfilgrastim was reported to decrease $\mathrm{FN}$-related hospitalizations by more than $90 \%$; however, in our study, the difference in hospitalizations was 47 vs. $17 \%$ with the use of filgrastim and pegfilgrastim, respectively ${ }^{1}$. However, the overall cost of care was reduced with pegfilgrastim prophylactic administration, owing to savings associated with the decrease in FN events and in the FN degree of severity, with the reduction in the number of bed-days, including admission to the emergency department, management in different hospital departments such as infectology, intermediate therapy, or pediatric intensive therapy, laboratory and imaging studies, as well as the use of broad spectrum antimicrobials and transfusions and their efficacy, with efficacy being regarded as hematological recovery, taking into account both neutrophil and monocyte

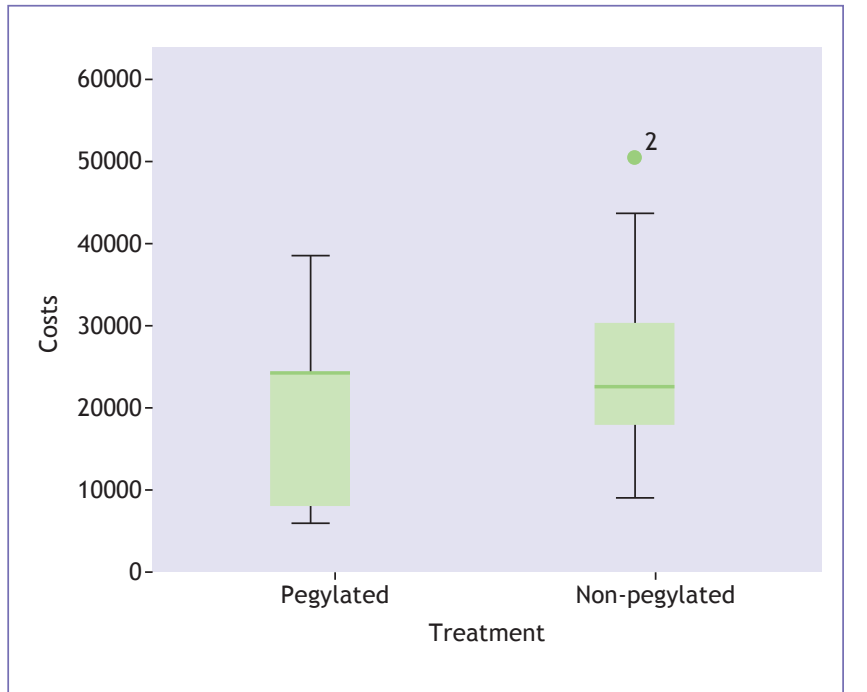

Figure 2. Global cost of febrile neutropenia prophylaxis with filgrastim and pegfilgrastim in pediatric patients with solid tumors (Mexican pesos)

recovery ${ }^{20}$. Pegfilgrastim added cost is approximately $\$ 22,000$ per vial in comparison with the filgrastim cost of $\$ 1,200$; however, the latter is used for 10 days, which leads to a total cost of $\$ 18,000$ per chemotherapy course, with a $25 \%$ saving. However, this saving is not reflected in the number of FN events and their severity or in hospitalization costs. Another important benefit found with the use of pegfilgrastim is that, owing to the decrease in FN events and faster recovery, patients do not delay the next chemotherapy course, which may be reflected on survival, as has been

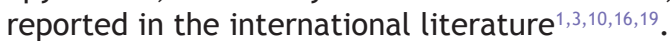

Currently, there are few pharmacoeconomic studies available, and they are highly important, since they should serve as a tool for decision making. According to some studies, the use of the pegylated form has been found to be more efficacious than filgrastim, owing to a decrease in both costs and FN events ${ }^{1-16}$. The cost-efficacy analysis looking at life-years gained in survivor pediatric patients treated with filgrastim vs. pegfilgrastim is the subject of a new trial that has not yet concluded; however, it could be inferred that long-term efficacy will be demonstrated.

\section{CONCLUSIONS}

Febrile neutropenia prophylaxis with pegfilgrastim has equale clinical and economic benefits as in adult patients; however, quality-adjusted life-years gained could be much higher in pediatric patients. The performance of pharmacoeconom ic studies in our country is of vital importance in order to select the best treatment for our patients, while providing greater benefits.

\section{DECLARATION OF INTEREST}

There is no conflict of interests since the study was NOT financed by the pharmaceutical industry. The costs related to this study were entirely financed by our institution. 


\section{REFERENCES}

1. Eldar-Lissai A, Cosler LE, Culakova E, Lyman GH. Economic analysis of prophylactic pegfilgrastim in adult cancer patients receiving chemotherapy. Value Health. 2008;11:272-9.

2. Aapro M, Crawford J, Kamioner D. Prophylaxis of chemotherapy-induced febrile neutropenia with granulocyte colony-stimulating factors: where are we now? Support Care Cancer. 2010;18:529-41.

3. Cooper KL, Madan J, Whyte S, Stevenson MD, Akehurst RL. Granulocyte colony-stimulating factors for febrile neutropenia prophylaxis following chemotherapy: systematic review and meta-analysis. BMC Cancer. 2011;11:404.

4. Kuderer NM, Dale DC, Crawford J, Cosler LE, Lyman GH. Mortality, morbidity and cost associated with febril neutropenia in adult cancer patients. Cancer. 2006;106:2258-66.

5. Talcott JA, Whalen A, Clark J, Rieker PP, Finberg R. Home antibiotic therapy for low-risk cancer patients with fever and neutropenia: a pilot study of 30 patients based on a validated prediction rule. J Clin Oncol. 1994;12:107-14.

6. Weycker D, Malin J, Barron R, Edelsberg J, Kartashov A, Oster G. Comparative effectiveness of filgrastim, pegfilgrastim, and sargramostim as prophylaxis against hospitalization for neutropenic complications in patients with cancer receiving chemotherapy. Am J Clin Oncol. 2012;35:267-74.

7. Caggiano V, Weiss RV, Richert TS, et al. Incidence, cost and mortality of neutropenia hospitalization associated whit chemotherapy. Cancer. 2008:103:1916-24.

8. Renwick W, Pettengell R, Green M. Use of filgrastim and pegfilgrastim to support delivery of chemotherapy: twenty years of clinical experience. BioDrugs. 2009;23:175-86.

9. Aapro MS, Cameron DA, Pettengell R, et al. EORTC guidelines for the use of granulocyte colony stimulating factor to reduce the incidence of chemotherapy-induced febrile neutropenia in adult patients with lymphoproliferative disorders and solid tumours. Eur $\mathrm{J}$ Cancer. 2006;42:2433-53.

10. Smith TJ, Khatcherssian J, Lyman GH, et al. Update of recommendations for the use of with blood cell growth factors: an evidence-based clinical practice guideline. J Clin Oncol. 2006;24:3187-205.

11. Lyman G, Lalla A, Barron R, Dubois RW. Cost-effectiveness of pegfilgrastim versus 6-day filgrastim primary prophylaxis in patients with non-Hodgkin's lymphoma receiving CHOP-21 in United States. Curr Med Res Opin. 2009;25:401-11.

12. Talcott JA, Whalen A, Clark J, Rieker PP, Finberg R. Home antibiotic therapy for low-risk cancer patients with fever and neutropenia: a pilot study of 30 patients based on a validated prediction rule. J Clin Oncol. 1994;12:107-14.

13. Lui Z, Doan QV, Malin J, et al. The economic value of primary prophylaxis using pegfilgrastim compared with filgrastim in patients with breast cancer in the UK. Appl Health Econ Health Policy. 2010;7:193-205.

14. Whyte S, Cooper KL, Stevenson MD, Madan J, Akehurst R. Cost-effectiveness of granulocyte colony-stimulating factor prophylaxis for febrile neutropenia in breast cancer in the United Kingdom. Value Health. 2011;14:465-74.

15. Sehouli J, Goertz A, Steinle T, et al. [Pegfilgrastim vs filgrastim in primary prophylaxis of febrile neutropenia in patients with breast cancer after chemotherapy: a cost-effectiveness analysis for Germany]. Dtsch Med Wochenschr. 2010;135:385-9.

16. Ishiguro $\mathrm{H}$, Kondo $M$, Hoshi SL, et al. Economic evaluation of intensive chemotherapy with prophylactic granulocyte colony-stimulating factor for patients with high-risk early breast cancer in Japan. Clin Ther. 2010;32:311-26.
17. Aapro MS, Bohlius J, Cameron DA, et al. 2010 update of EORTC guidelines for the use of granulocyte-colony stimulating factor to reduce the incidence of chemotherapy-induced febrile neutropenia in adult patients with lymphoproliferative disorders and solid tumors. Eur J Cancer. 2011;47:8-32.

18. Weycker D, Malin J, Kim J, et al. Risk of hospitalization for neutropenic complications of chemotherapy in patients with primary solid tumors receiving pegfilgrastim or filgrastim prophylaxis: a retrospective cohort study. Clin Ther. 2009;31:1069-81.

19. Crawford J, Dale DC, Kuderer NM, et al. Risk and timing of neutroper nic events in adult cancer patients receiving chemotherapy: the results of a prospective nationwide study of oncology practice. J Natl Compro Canc Netw. 2008;6:109-18.

20. Scholz M, Ackermann M, Emmrich F, Loeffler M, Kamprad M. Effec tiveness of cytopenia prophylaxis for different filgrastim and pegfilgrastim schedules in a chemotherapy mouse model. Biologics. 2009; 3 : 27-37.

21. Johnston E, Crawford J, Blackwell S, et al. Randomized, dose-escala tion study of SD/01 compared with daily filgrastim in patients receiving chemotherapy. J Clin Oncol. 2000;18:2522-8.

22. Lyman GH, Kuderer N, Greene J, Balducci L. The economics of febrile neutropenia: implications for the use of colony-stimulating factors. Eur J Cancer. 1998;34:1857-64.

23. Hirsch BR, Lyman GH. Pharmacoeconomics of the myeloid growth factors: a critical and systematic review. Pharmacoeconomics. 2012; 30:497-511.

24. Colester LE, Sivasubramaniam V, Agboola O, et al. Effect of outpatient treatmen of febrile neutropenia on the risk threshold for the use of CSF in patients with cancer treated with chemotherapy. Value Health. 2005;8:47-52.

25. Hershman DL, Wilde ET, Wright JD, et al. Uptake and economic impact of first-cycle colony-stimulating factor use during adjuvant treatment of breast cancer. J Clin Oncol. 2012;30:806-12.

26. Timmer-Bonte JN, Andag EM, Smit HJ, et al. Cost-effectiveness of adding granulocyte colony-stimulating factor to primary prophylaxis with antibiotics in small-cell lung cancer. J Clin Oncol. 2006;24: 2991-7.

27. Costa LJ, Kramer C, Hogan KR, et al. Pegfilgrastim vs filgrastim based autologous hematopoietic stem cell mobilization in the setting of preemptive use of plerixafor: efficacy and cost analysis. Transfusion. 2012;52:2375-81.

28. Sebban C, Lefranc A, Perrier L, et al. A randomised phase II study of the efficacy, safety and cost-effectiveness of pegfilgrastim and filgrastim after autologous stem cell transplant for lymphoma and myeloma (PALM study). Eur J Cancer. 2012;48:713-20.

29. Wannesson L, Luthi F, Zucca E, et al. Pegfilgrastim to accelerate neuz trophil engraftment following peripheral blood stem cell transplant and reduce the duration of neutropenia, hospitalization, and use of intravenous antibiotics: a phase II study in multiple myeloma and lymphoma and comparison with filgrastim-treated matched controls. Leuk Lymphoma. 2011;52:436-43.

30. Aapro M, Cornes P, Abraham I. Comparative cost-efficiency across the European G5 countries of various regimens of filgrastim, biosimilar filgrastim, and pegfilgrastim to reduce the incidence of chemotherapy-induced febrile neutropenia. J Oncol Pharm Pract. 2012;18:171-9.

31. Crokaert F. Febrile neutropenia in children. Klinische Padiatrie. 2008; (Suppl 1):S9-16.

32. Fox E, Widemann B, Hawkins DS, et al. Randomized trial and pharmacokinetic study of pegfilgrastim vs. filgrastim after dose-intensive chemotherapy in young adults and children with sarcomas. Clin Cancer Res. 2009;15:7361-7. 